\title{
Disinfection effect of adding slightly acidic electrolyzed water to artificial seawater under the condition of static hybrid
}

\author{
Chunfang Wang ${ }^{1,2}$, Xiaoling Huang ${ }^{1}$, Shuo Wang ${ }^{1}$, Yong $\mathrm{Yu}^{1}$, Songming $\mathrm{Zhu}^{1,3}$, Zhangying $\mathrm{Ye}^{1,3^{*}}$ \\ (1. College of Biosystems Engineering and Food Science, Zhejiang University, Hangzhou 310058, China; \\ 2. Research Center for Agricultural Products Preservation and Processing, Shanghai Academy of Agricultural Sciences, \\ Shanghai 201403, China; \\ 3. Ocean Academy, Zhejiang University, Zhoushan 316000, China)
}

\begin{abstract}
Mixed solution of slightly acidic electrolyzed water (SAEW) and artificial seawater was used to investigate the disinfection potential of SAEW in artificial seawater. Inoculated Vibrio parahaemolyticus (suspended in 3\% sodium chloride alkaline peptone water and $0.85 \%$ sodium chloride water, respectively) was subjected to different mixed-SAEW and SAEW immersion treatments $(5-20 \mathrm{mg} / \mathrm{L}$ available chlorine concentration (ACC)). In the presence of organic matter, $4.07 \log \mathrm{CFU} / \mathrm{mL}$ significant reduction $(p<0.05)$ was achieved after treating with $20 \mathrm{mg} / \mathrm{L}$ mixed-SAEW for $15 \mathrm{~min}$. There was $5.13 \log \mathrm{CFU} / \mathrm{mL}$ reduction after treating with $15 \mathrm{mg} / \mathrm{L}$ SAEW for $15 \mathrm{~min}$. For $V$. parahaemolyticus suspended in $0.85 \%$ sodium chloride solution, it was undetected after $30 \mathrm{~s}$ SAEW treatment $(5 \mathrm{mg} / \mathrm{L} \mathrm{ACC})$ or $120 \mathrm{~s}$ mixed-SAEW treatment $(10 \mathrm{mg} / \mathrm{L}$ ACC). At a ratio of SAEW and artificial seawater at 1:15 (V/V), SAEW could inactivate $V$. parahaemolyticus to undetectable level in artificial seawater in one minute, which was comparable with UV treatment of $10 \mathrm{~W}$. The results indicated high sanitization potential of SAEW against $V$. parahaemolyticus in aquaculture seawater.
\end{abstract}

Keywords: slightly acidic electrolyzed water, disinfection, inactivation, Vibrio parahaemolyticus, artificial seawater, ultraviolet DOI: $10.25165 /$ j.ijabe.20201302.4194

Citation: Wang C F, Huang X L, Wang S, Yu Y, Zhu S M, Ye Z Y. Disinfection effect of adding slightly acidic electrolyzed water to artificial seawater under the condition of static hybrid. Int J Agric \& Biol Eng, 2020; 13(2): 218-222.

\section{Introduction}

Aquaculture is one of the fastest-growing food producing sectors, due to increasing demand and environmental considerations. Global aquaculture has grown dramatically over the past 50 years to 80 million tonnes, accounting for around $46 \%$ of the world's fish food supply ${ }^{[1]}$. As water is filled in the place where fish and other aquatic product live in, water quality is significantly important. Vibrio parahaemolyticus, halophilic bacteria, is a prevalent food-borne pathogen and exists in a variety of raw seafood, such as oyster and shrimp. Consumption of raw or undercooked seafood contaminated with $V$. parahaemolyticus may cause gastrointestinal episodes ${ }^{[2-4]}$. Therefore, elimination of $V$. parahaemolyticus at the origin of aquaculture system is of great importance. However, with consumers' increasing consciousness of food safety, it requires aquaculture free from the use of chemicals such as antibiotics. Therefore, developing eco-friendly disinfectant is necessary and promising to replace chemicals.

Slightly acidic electrolyzed water (SAEW) becomes a research focus in recent years as an effective, nontoxic and environmentally

\section{Received date: 2018-02-24 Accepted date: 2019-12-23}

Biographies: Chunfang Wang, PhD, Research assistant, research interests: agricultural engineering, Email: fhwcf@126.com; Xiaoling Huang, MS candidate, research interest: aquacultural engineering, Email: biglingling@ yeah.net; Shuo Wang, $\mathrm{PhD}$ candidate, research interest: aquacultural engineering, Email: sywshuo@163.com; Yong Yu, Associate Professor, research interest: agricultural engineering, Email: yyuzju@zju.edu.cn; Songming Zhu, Professor, research interests: agricultural engineering and aquacultural engineering, Email: zhusm@zju.edu.cn.

*Corresponding author: Zhangying Ye, Professor, research interest: agricultural engineering and aquacultural engineering. College of Biosystems Engineering and Food Science, Zhejiang University, Hangzhou 310058, China. Tel: +86-15168310563, Email: yzyzju@zju.edu.cn. friendly disinfectant with high bactericidal effect ${ }^{[5]}$. It is also known as weakly acidic electrolyzed water (WAEW). It was reported that SAEW may have equivalent or higher bactericidal activity than $\mathrm{NaClO}$ solution with lower available chlorine concentration $(\mathrm{ACC})^{[6-9]}$. As an effective disinfectant, many studies have demonstrated the high bactericidal efficacy of SAEW against Escherichia coli, Staphylococcus aureus, Salmonella spp., Vibrio vulnificus and Vibrio parahaemolyticus ${ }^{[9-11]}$. SAEW contains a high concentration (approximately 95\%) of hypochlorous acid ${ }^{[12,13]}$, which is more effective as a sanitizing agent than an equivalent concentration of the hypochlorite ion ${ }^{[14]}$. Furthermore, with a near neutral $\mathrm{pH}$ of 5.0-6.5, it helps reduce the risk of human health and safety issues from $\mathrm{Cl}_{2}$ off-gassing and decrease the risk of corrosion ${ }^{[15]}$.

There are many studies focused on the effect of acidic electrolyzed water (AEW, $\mathrm{pH}$ 2.3-2.7) or SAEW on seafood. As for the use of SAEW in purification of marine aquaculture, electrolyzation of seawater directly without a membrance was used to obtain the electrolyzed water for the disinfection in aquaculture $^{[16-18]}$. Katayose et al. ${ }^{[18]}$ reported that electrolyzed natural seawater is safe and effective with ACC at an adequate level in aquaculture. Jorquera et al. ${ }^{[17]}$ reported that electrolysis between 1.0-4.0 A was able to disinfect safely and electrolyzed seawater achieved higher microalga growth than traditionally autoclaved seawater or seawater treated with germicidal ultraviolet light (UV). It was reported that $0.2 \mathrm{mg} / \mathrm{L}$ of chlorine was safe for oysters and after depuration with electrolyzed seawater for $24 \mathrm{~h}, E$. coli counts in artificially contaminated oysters decreased to detection limit $^{[16]}$. Seawater, especially natural seawater, has many ions, which may cause corrosion to the electrolysis device or react with oxide. Therefore, instead of electrolyzing seawater, adding SAEW directly into seawater to inactivate $V$. 
parahaemolyticus is considerable. Several studies have demonstrated the efficacy of killing $V$. parahaemolyticus by WAEW or AEW in vitro ${ }^{[9,19]}$. However, there are fewer studies about the effect of electrolyzed seawater on $V$. parahaemolyticus and researches related to the inactivation of $V$. parahaemolyticus in seawater using SAEW are even much scarcer.

Although some studies have been performed to study the electrolyzed seawater, no studies were reported on using SAEW to disinfect seawater. Therefore, the objects were: 1 ) to evaluate the bactericidal effect of SAEW diluted by distilled water and artificial seawater on $V$. parahaemolyticus with and without organic contents; 2) to determine the efficiency of SAEW for the elimination of $V$. parahaemolyticus in artificial seawater and compared with UV treatment.

\section{Materials and methods}

\subsection{Bacterial culture and preparation of inoculums}

The culture of $V$. parahaemolyticus (ATCC 33847) was obtained from China General Microbiological Culture Collection Center (CGMCC, Beijing, China). The culture was transferred on basis every 2 weeks to ensure their viability. To prepare the inoculation stock, several loops of isolated colonies of stock culture were transferred to $100 \mathrm{~mL}$ sterile $3 \%$ sodium chloride alkaline peptone water ( $30 \mathrm{~g} / \mathrm{L}$ sodium chloride, $10 \mathrm{~g} / \mathrm{L}$ peptone, $\mathrm{pH} 8.0 \pm 0.5$ ) in $250 \mathrm{~mL}$ Erlenmeyer flasks and incubated at $37^{\circ} \mathrm{C}$ for $24 \mathrm{~h}$ with agitation $(150 \mathrm{r} / \mathrm{min})$. Following incubation, $30 \mathrm{~mL}$ inoculation stock was transferred into a $50 \mathrm{~mL}$ sterilized centrifuge tube and centrifuged at $3200 \mathrm{~g}$ for $10 \mathrm{~min}$ at $20^{\circ} \mathrm{C}(5810 \mathrm{R}$, Eppendorf $\mathrm{AG}$, Germany). The cell pellet was washed and resuspended in $30 \mathrm{~mL}$ $3 \%$ sodium chloride solution peptone water or $0.85 \%$ sodium chloride solution, respectively. Populations in individual cultures were determined by spread plating $0.1 \mathrm{~mL}$ portions of appropriately diluted cultures on thiosulfate citrate bile sucrose agar (TCBS) plates, respectively, and incubation at $37^{\circ} \mathrm{C}$ for $24 \mathrm{~h}$ before counting. The initial $V$. parahaemolyticus inoculation was approximately $10^{8}$ to $10^{9}$ colony forming units (CFU)/mL.

\subsection{Preparation of treatment solutions}

SAEW was produced by electrolysis of $6 \% \mathrm{HCl}$ solution using a flow-type electrolysis apparatus (Figure 1) equipped with an electrolytic cell without a separating membrane between anode and cathode at a voltage of $220 \mathrm{~V}$ (HD-240L, Wangpu Trading Co., Ltd, Shanghai, China). The SAEW was produced immediately prior to use for evaluating its bactericidal activities and SAEW with $\mathrm{pH}$ $\sim 6.0$, oxidation reduction potential (ORP) of $\sim 880 \mathrm{mV}$ and ACC of $\sim 30 \mathrm{mg} / \mathrm{L}$ was collected for the experiments.

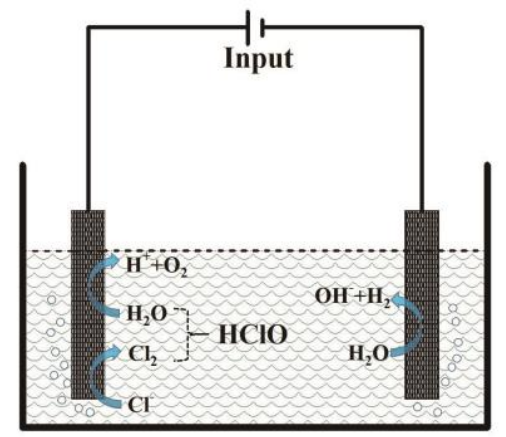

Figure 1 Schematic diagram of the electrolysis apparatus

The treatment SAEW solutions at low levels of ACC were diluted using distilled water prior to measurement. To investigate the inactivation ability of SAEW in seawater, concentrated artificial seawater was also used to dilute initial SAEW and these solutions called mixed-SAEW. The formula of artificial seawater was modified according to Berges et al. ${ }^{[20]}$ and s shown in Table 1. The final salinity of the artificial seawater was $3.3 \% \pm 0.1 \%$ and $\mathrm{pH}$ was $8.00 \pm 0.10$.

Table 1 Formula of artificial seawater

\begin{tabular}{cccc}
\hline Solution I $^{\text {a }}$ & Concentration $/ \mathrm{g} \cdot \mathrm{L}^{-1}$ & Solution II & Concentration $/ \mathrm{g} \cdot \mathrm{L}^{-1}$ \\
\hline $\mathrm{NaCl}$ & 21.19 & $\mathrm{MgCl}_{2} \cdot 6 \mathrm{H}_{2} \mathrm{O}$ & 9.592 \\
$\mathrm{Na}_{2} \mathrm{SO}_{4}$ & 3.55 & $\mathrm{CaCl}_{2} \cdot 2 \mathrm{H}_{2} \mathrm{O}$ & 1.344 \\
$\mathrm{KCl}$ & 0.599 & $\mathrm{SrCl}_{2} \cdot 6 \mathrm{H}_{2} \mathrm{O}$ & 0.0218 \\
$\mathrm{NaHCO}_{3}$ & 0.174 & & \\
$\mathrm{KBr}$ & 0.0863 & & \\
$\mathrm{H}_{3} \mathrm{BO}_{3}$ & 0.023 & \\
$\mathrm{NaF}^{a}$ & 0.0028 & \\
\hline Note: & & \\
\hline
\end{tabular}

\subsection{Determination of ORP, $\mathrm{pH}$ and ACC of SAEW}

The ORP, $\mathrm{pH}$ and ACC of treatment solutions were measured in triplicate immediately after preparation and before the bactericidal experiments. The $\mathrm{pH}$ and ORP values of SAEW were measured with a dual scale pH/ORP meter (Mettler-Toledo International Co., Ltd., Zurich, Switzerland). The ACC in SAEW was determined by a colorimetric method using a digital chlorine test kit (RC-3F, Kasahara Chemical Instruments Co., Ltd., Saitama, Japan).

\subsection{Mixed-SAEW and SAEW treatments of $V$. parahaemolyticus}

Bacterial stocks approximately $8 \log \mathrm{CFU} / \mathrm{mL}$ were prepared as previously described in section 2.1. For $V$. parahaemolyticus resuspended in sterilized 3\% sodium chloride solution peptone water, one milliliter of the prepared $V$. parahaemolyticus culture solution was added into $9 \mathrm{~mL}$ of mixed-SAEW or SAEW with different ACC (treatment) and $0.85 \%$ sodium chloride solution (control). A timer was started and the tube was mixed immediately at $(23 \pm 2)^{\circ} \mathrm{C}$. At the end of each contact time, $1 \mathrm{~mL}$ of each sample was transferred to a tube containing $9 \mathrm{~mL}$ of sterilized neutralizer $(0.5 \%$ sodium thiosulphate, $0.85 \%$ sodium chloride solution) followed by serial 10 -fold dilution with $0.85 \%$ sodium chloride solution dilution blanks. Surviving population in each culture was confirmed by plating $0.1 \mathrm{~mL}$ portions of appropriately diluted cultures on TCBS plates in triplicate, respectively. Colonies of survived $V$. parahaemolyticus were enumerated on TCBS plates after incubation at $37^{\circ} \mathrm{C}$ for $24 \mathrm{~h}$. The reduction of $V$. parahaemolyticus was expressed as $\log \left(N_{0} / N_{t}\right)$, where $N_{0}$ is the count before treatment and $N_{t}$ the count after treatment.

As for $V$. parahaemolyticus resuspended in $0.85 \%$ sodium chloride solution, one milliliter of the prepared $V$. parahaemolyticus was added into $9 \mathrm{~mL}$ of mixed-SAEW with different ACC (treatment), and the tube was mixed immediately at $(23 \pm 2)^{\circ} \mathrm{C}$. At the end of each contact time $(30 \mathrm{~s}, 60 \mathrm{~s}$ and $90 \mathrm{~s})$, $1 \mathrm{~mL}$ of each sample was transferred to a sterilized tube containing $9 \mathrm{~mL}$ of $3 \%$ sodium chloride solution peptone water and sealed with a silica gel stopper. Then incubate at $37^{\circ} \mathrm{C}$ for $48 \mathrm{~h}$ with agitation $(150 \mathrm{r} / \mathrm{min})$. This is an enrichment to see visible growth of survived and injured $V$. parahaemolyticus. Tubes with negative growth were considered that $V$. parahaemolyticus in treated samples was completely inactivated.

\subsection{SAEW treatment of $V$. parahaemolyticus in artificial seawater}

Five milliliters of cultured $V$. parahaemolyticus stock was added into $245 \mathrm{~mL}$ and $220 \mathrm{~mL}$ artificial seawater in $500 \mathrm{~mL}$ bake 
with 1 min stirring and initial count was $7.60 \pm 0.04 \log C F U / m L$. Then a volume of $25 \mathrm{~mL}, 15 \mathrm{~mL}, 10 \mathrm{~mL} \mathrm{SAEW} \mathrm{(ACC=30} \mathrm{mg/L,}$ $\mathrm{ORP}=858 \pm 2 \mathrm{mV}, \mathrm{pH}=5.69 \pm 0.04)$ was added into the contaminated artificial seawater $(250 \mathrm{~mL}, 225 \mathrm{~mL}$ and $250 \mathrm{~mL}$, respectively) and the proportion was 1:10, 1:15 and 1:25 (V/V), respectively. At the end of each contact time, $1 \mathrm{~mL}$ of each sample was transferred to a tube containing $9 \mathrm{~mL}$ of neutralizer $(0.5 \%$ sodium thiosulphate, $0.85 \%$ sodium chloride solution) followed by serial 10 -fold dilution in $0.85 \%$ sodium chloride solution dilution blanks. Surviving population in each culture was confirmed by plating $0.1 \mathrm{~mL}$ portions of appropriately diluted cultures on TCBS plates in triplicate, respectively. Colonies of survived $V$. parahaemolyticus were enumerated on TCBS plates after incubation at $37^{\circ} \mathrm{C}$ for $24 \mathrm{~h}$. In the meantime, $1 \mathrm{~mL}$ of each sample was also transferred to a tube containing $9 \mathrm{~mL}$ of $3 \%$ sodium chloride alkaline peptone water and sealed with a silica gel stopper. Then incubate at $37^{\circ} \mathrm{C}$ for $48 \mathrm{~h}$ with agitation $(150 \mathrm{r} / \mathrm{min})$, which is the same enrichment step as section 2.4 described.

\subsection{UV treatment of $\boldsymbol{V}$. parahaemolyticus in artificial seawater}

Different power of UV light $(10 \mathrm{~W}$ and $15 \mathrm{~W})$ was used to inactivate $V$. parahaemolyticus in artificial seawater. UV light was set into the baker containing $250 \mathrm{~mL}$ artificial seawater $(6.55 \pm 0.26 \log \mathrm{CFU} / \mathrm{mL})$. The enumeration and enrichment steps were the same as described in section 2.5 .

\subsection{Data analysis}

Statistical analysis was performed using one-way analysis of variance (ANOVA). Duncan test $(p<0.05)$ was applied to compare the average values using SPSS 20.0 (Statistical Package for the Social Sciences).

\section{Results and discussion}

3.1 Inactivation effect of mixed-SAEW and SAEW on $V$. parahaemolyticus in broth

The results in Figure 2 show reduced $V$. parahaemolyticus when being suspended in $3 \%$ sodium chloride alkaline peptone water $(0.1 \%$ peptone $)$ treated by mixed-SAEW and SAEW solutions. The physicochemical properties of mixed-SAEW and SAEW were attached in the figure. The initial concentration of $V$. parahaemolyticus was $\sim 8 \log \mathrm{CFU} / \mathrm{mL}$.

We can see from Figure 2 that reductions of $V$. parahaemolyticus significantly increased with increasing exposure time (except for $10 \mathrm{mg} / \mathrm{L}$ at $5 \mathrm{~min}$ and $10 \mathrm{~min}$ ). The reductions of $V$. parahaemolyticus were $1.63 \log \mathrm{CFU} / \mathrm{mL}, 2.46 \log \mathrm{CFU} / \mathrm{mL}$ and $2.25 \log \mathrm{CFU} / \mathrm{mL}$ after $5 \mathrm{~min}$ treatment of mixed-SAEW with ACC $10 \mathrm{mg} / \mathrm{mL}, 15 \mathrm{mg} / \mathrm{mL}$ and $20 \mathrm{mg} / \mathrm{mL}$, respectively. It increased to $4.07 \log \mathrm{CFU} / \mathrm{mL}$ after treating with $20 \mathrm{mg} / \mathrm{mL}$ ACC of mixed-SAEW for $15 \mathrm{~min}$. As for samples treated by SAEW, reductions of $V$. parahaemolyticus also significantly increased with increasing treating time and ACC level. It increased from $0.65 \pm 0.05 \log \mathrm{CFU} / \mathrm{mL}$ at $5 \mathrm{mg} / \mathrm{L} \mathrm{ACC}, 5 \mathrm{~min}$ to $5.13 \pm 0.03$ $\log \mathrm{CFU} / \mathrm{mL}$ at $15 \mathrm{mg} / \mathrm{L} \mathrm{ACC}, 15 \mathrm{~min}$.

Tables attached in Figure 2 illustrate the properties of mixed-SAEW and SAEW. As the ACC of initially generated SAEW was approximate $30 \mathrm{mg} / \mathrm{L}$, the ACC values of diluted mixed-SAEW and SAEW were ranged from 5 to $20 \mathrm{mg} / \mathrm{L}$. These diluted mixed-SAEW solutions had a $\mathrm{pH}$ value ranging between 6.83 and 6.91, and an ORP value ranging from 844 to $867 \mathrm{mV}$, whereas, it was 6.17 to 6.22 and 865 to $893 \mathrm{mV}$ for diluted SAEW solutions. When ACC was proportionally decreased as a result of dilution by artificial seawater and distilled water, ORP decreased and $\mathrm{pH}$ increased but not proportionally. However, the inactivation effect of SAEW treatment increased with treating time and ACC level, which demonstrated that ACC is the main factor for the inactivation. It was consistent to other studies that the surviving population of microorganisms decreased with the increase of exposure time and ACC level ${ }^{[9-11]}$. For mixed-SAEW treated samples, the reduction of $V$. parahaemolyticus had no significant difference between $20 \mathrm{mg} / \mathrm{mL}$ ACC, $10 \mathrm{~min}$ and $15 \mathrm{mg} / \mathrm{mL}$ ACC, $10 \mathrm{~min}$. This may be due to the combination effect of ACC and ORP. Huang et al. ${ }^{[21]}$ reported that +200 to $+800 \mathrm{mV}$ was the best ORP extent for aerobic microorganisms. High ORP could cause the modification of metabolic fluxes and ATP production due to the change in the electron flow in cells ${ }^{[9]}$. Therefore, similar reductions were obtained by mixed-SAEW $15 \mathrm{mg} / \mathrm{L}$ and $20 \mathrm{mg} / \mathrm{L} \mathrm{ACC}$ with similar ORP of $863 \mathrm{mV}$ and $867 \mathrm{mV}$. Whereas, diluted SAEW does not only reduce the levels of ACC but also its physico properties. The value of ORP was the ratio of oxidized to reduced species in solution ${ }^{[22]}$. Though the decrease of ORP was not proportional, the dilution did decrease the concentration of oxide in solution and lowered the inactivation effect $^{[23]}$. This might be the reason that the reduction differences between $15 \mathrm{mg} / \mathrm{L} \mathrm{ACC}$ and $20 \mathrm{mg} / \mathrm{L}$ ACC were much lower than those between 10 and $15 \mathrm{mg} / \mathrm{L} \mathrm{ACC}$, since ORP also plays an important role in inactivation combined with ACC.
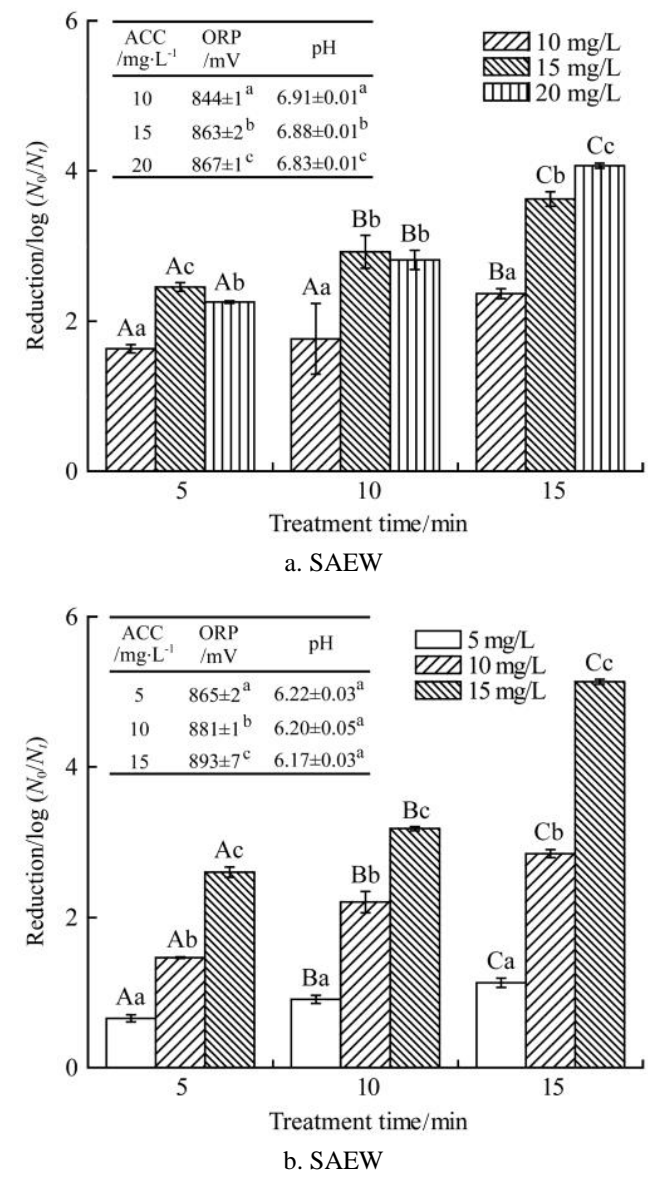

Note: ORP and $\mathrm{pH}$ of mixed-SAEW and SAEW were attached respectively in the figure and different letters $(a, b, c)$ indicate significant difference $(p<0.05)$ in each column. Vertical bars represent $\pm \mathrm{SD}, n=3$. Different letters $(\mathrm{a}, \mathrm{b}, \mathrm{c})$ above the bars indicate significant difference $(p<0.05)$ between different ACC at the same treating time, while A, B, C indicate significant difference $(p<0.05)$ between different treating times at the same ACC.

Figure 2 Reduction results of $V$. parahaemolyticus treated by mixed-SAEW and SAEW

Comparing between SAEW and mixed-SAEW (with the same ACC of 10 and $15 \mathrm{mg} / \mathrm{L}$ ), SAEW showed better inactivation effect 
than mixed-SAEW. The reductions were 2.85 and 5.14 $\log \mathrm{CFU} / \mathrm{mL}$ after treating $15 \mathrm{~min}$ with $10 \mathrm{mg} / \mathrm{mL}$ and $15 \mathrm{mg} / \mathrm{mL}$ ACC of SAEW respectively, while they were $2.37 \log \mathrm{CFU} / \mathrm{mL}$ and $3.63 \log \mathrm{CFU} / \mathrm{mL}$ for the same treating time and ACC of mixed-SAEW. These differences may mainly caused by different pH. $\quad$. parahaemolyticus is sensitive to acid and its optimal growing $\mathrm{pH}$ condition was ranging from 6.5 to 8.5. Due to the mixing with artificial seawater, the $\mathrm{pH}$ values of mixed-SAEW (approaching 7) were higher than SAEW (approaching 6). Low $\mathrm{pH}$ may sensitize the outer membrane of bacterial cells to the entry of hypochlorous acid into bacterial cells ${ }^{[24]}$. On the other hand, the salinity of SAEW was zero, which is an unfavorable growth condition for $V$. parahaemolyticus. Bacteria are more resistance under ideal conditions. This may be the main reason that reductions in SAEW treated samples were higher than those in mixed-SAEW.

The inactivation effect of $V$. parahaemolyticus mainly increased with increasing ACC of both mixed-SAEW and SAEW. Though the inactivation effect of mixed-SAEW was lower than SAEW, it was efficient to inactivate $V$. parahaemolyticus with the presence of organic matter. As an effective, nontoxic and environmentally friendly disinfectant with high bactericidal effect, SAEW has large potential in practical use.

\subsection{Inactivation of mixed-SAEW on $V$. parahaemolyticus without organic matter}

Although many researchs have revealed the efficiency of SAEW to inactivate pure microb, we need to confirm the effect of mixed-SAEW on pure $V$. parahaemolyticus. Table 2 shows the enrichment results of $V$. parahaemolyticus suspended in $0.85 \%$ sodium chloride solution after being treated with different mixed-SAEW at different times, and corresponding physicochemical properties of mixed-SAEW are also shown in the table. These diluted mixed-SAEW solutions had a $\mathrm{pH}$ value ranging between 6.90 and 7.08, and an ORP value ranged from 746 to $856 \mathrm{mV}$.

Table 2 The Vibrio parahaemolyticus destruction and physicochemical properties of different mixed-SAEW

\begin{tabular}{cccccc}
\hline $\mathrm{ACC} / \mathrm{mg} \cdot \mathrm{L}^{-1}$ & $30 \mathrm{~s}$ & $60 \mathrm{~s}$ & $120 \mathrm{~s}$ & $\mathrm{ORP} / \mathrm{mV}$ & $\mathrm{pH}$ \\
\hline 20 & $+^{\mathrm{A}}$ & $-^{\mathrm{B}}$ & - & $856 \pm 4^{\mathrm{aC}}$ & $6.90 \pm 0.03^{\mathrm{a}}$ \\
15 & + & - & - & $811 \pm 3^{\mathrm{b}}$ & $7.02 \pm 0.02^{\mathrm{b}}$ \\
10 & + & + & - & $772 \pm 4^{\mathrm{c}}$ & $7.00 \pm 0.05^{\mathrm{b}}$ \\
5 & + & + & + & $746 \pm 6^{\mathrm{d}}$ & $7.08 \pm 0.03^{\mathrm{b}}$ \\
\hline
\end{tabular}

Note: ${ }^{\mathrm{A}}$ Negative for culture without visible growth, means completely
inactivated. ${ }^{\mathrm{B}}$ Positive for culture with visible growth, means not completely inactivated. $\quad{ }^{\mathrm{C}}$ Different letters $(\mathrm{a}, \mathrm{b}, \mathrm{c}, \mathrm{d})$ indicate significant difference $(p<0.05)$ in each column.

The inactivation trend in $V$. parahaemolyticus under organic free condition was similar to the results shown in section 3.1, which the inactivation effect was increased with increasing treating time and ACC level. After $48 \mathrm{~h}$ incubation, positive growth of $V$. parahaemolyticus observed after 2 min treatment of mixed-seawater with $5 \mathrm{mg} / \mathrm{L}$ ACC. However, no growth of $V$. parahaemolyticus was observed after $2 \mathrm{~min}$ treatment with $10 \mathrm{mg} / \mathrm{L}$ ACC. Time for negative growth reduced to $60 \mathrm{~s}$ when ACC increased to $15 \mathrm{mg} / \mathrm{L}$ and $20 \mathrm{mg} / \mathrm{L}$. This indicates that SAEW was able to completely inactivate $V$. parahaemolyticus, but it had different minimum treating time with different ACC levels. The result was also consistent to the results in section 3.1 that the inactivation of SAEW was much more effective than mixed-SAEW. Negative growth was observed after $30 \mathrm{~s}$ under the SAEW treatment with $5 \mathrm{mg} / \mathrm{L}$ of ACC (data not shown). It was due to the higher ORP and lower $\mathrm{pH}$ of SAEW than mixed-SAEW (ORP $838 \mathrm{VS} 746 \mathrm{mV}$, pH $6.73 \mathrm{VS} 7.08$ at $5 \mathrm{mg} / \mathrm{mL}$ of ACC).

Quan et al. ${ }^{[9]}$ detected the minimum ACC of WAEW for bactericidal activity against cell suspensions of Vibrio vulnificus and $V$. parahaemolyticus, which was approximately $0.1 \mathrm{mg} / \mathrm{L}$ (volume ratio of cell suspension and WAEW was 1:19). However, for cell cultures (cell in the medium), minimum ACC was approximately $20 \mathrm{mg} / \mathrm{L}$. These differences were probably due to different kinds of electrolyzed water and different volume ratios. Basically, it was due to different values of ACC, ORP and $\mathrm{pH}$. These also confirmed the theory that the bactericidal effect of SAEW was the combined effect of ORP, ACC and $\mathrm{pH}$. What's more, even different strains of the same serotype can differ in their resistance towards an intervention ${ }^{[25]}$.

These results indicated that it took longer time and higher ACC to achieve better inactivation results when $V$. parahaemolyticus was suspended in broth than suspended in $0.85 \%$ sodium chloride solution. Organic matter would decrease bactericidal effect of SAEW. Current research reports showed that organic matter could react with free chlorine to generate the combined chlorine with low antiseptic effect, thus reduced the bactericidal effect ${ }^{[22,26]}$. The results in this paper were consistent with them. The inactivation effect increased with the increasing contacting time and the absence of organic matter, which is consisted with Issa-Zacharia et al. ${ }^{[10]}$

As an effective, nontoxic and environmentally friendly disinfectant with high bactericidal effect, SAEW has large potential in practical use. Therefore, it's necessary to choose an appropriate ACC and treating time to get expected destruction. In the absence of organic matter or low organic matter content, we can choose low ACC electrolysis water or reduce processing time, in order to reduce waste and improve efficiency.

\subsection{Inactivation effect of $V$. parahaemolyticus in artificial seawater by SAEW and UV}

Preliminary experiment indicated that $V$. parahaemolyticus could suvive for a long time in artificial seawater and the reduction was less than $1.5 \log C F U / m L$ after $10 \mathrm{~d}$ (data not shown). Previous results showed good inactivation effect of SAEW and mixed-SAEW on $V$. parahaemolyticus suspended in broth. The inactivation time and ACC level could reduce further when $V$. parahaemolyticus was suspended in $0.85 \%$ sodium chloride solution. We suppose it may have good sanitization potential as a kind of disinfectant additive to treat seawater.

Table 3 and 4 show the inactivation effect of SAEW and UV on $V$. parahaemolyticus inoculated in artificial seawater, according to the enumeration and enrichment results. The finial ACC values of different seawater with ratio of 1:10, 1:15 and 1:25 (V/V) was $3 \mathrm{mg} / \mathrm{L}, 2 \mathrm{mg} / \mathrm{L}$ and $1 \mathrm{mg} / \mathrm{L}$, respectively. When the ratio of SAEW and artificial seawater was 1:25(V/V), average count of $V$. parahaemolyticus enumeration was less than $10 \mathrm{CFU} / \mathrm{mL}$ and enrichment broth showed positive growth after 5 min treatment. Survival V. parahaemolyticus was detected even after $30 \mathrm{~min}$ (data not shown). However, they showed negative growth both on paltes and broth after $3 \mathrm{~min}$ and 1 min treatment when the ratios were $1: 15(\mathrm{~V} / \mathrm{V})$ and $1: 10(\mathrm{~V} / \mathrm{V})$, respectively.

While treated with UV of $10 \mathrm{~W}$, positive growth was observed both on enumeration plates and enrichment tubes after 5 min UV treatment. The results of $15 \mathrm{~W} \mathrm{UV}$ treatment were similar to SAEW treatment with a ratio of 1:15 that no $V$. parahaemolyticus colonies were formed on TCBS plates after $1 \mathrm{~min}$ treatment. 
Theoretically the ACC of 1:15 SAEW-seawater solution was approximately $1.9 \mathrm{mg} / \mathrm{L}$. In fact, it would be less than $1.9 \mathrm{mg} / \mathrm{L}$ due to the effect of dilution and inactivation, which is in the safe zone of $2.0 \mathrm{mg} / \mathrm{L}$ reported by Katayose et al. ${ }^{[18]}$

Therefore, with a ratio of 1:15 of SAEW and artificial seawater, SAEW was efficient and safe to inactivate $V$. parahaemolyticus in artificial seawater. It is promising to use SAEW as an addictive to treat seawater in aquaculture.

Table 3 Inactivation results of different SAEW ratio on V. parahaemolyticus in artificial seawater

\begin{tabular}{cccccccc}
\hline Ratio (V/V) & $1: 25$ & & \multicolumn{3}{c}{$1: 15$} & \multicolumn{2}{c}{$1: 10$} \\
\cline { 1 - 5 } Time & 5 min & & 1 min & 3 min & 5 min & 1 min & 3 min \\
\hline $\begin{array}{c}\text { Survivals } \\
/ \log \mathrm{CFU} \cdot \mathrm{mL}^{-1}\end{array}$ & $<1^{\mathrm{a}}$ & $<1$ & $0^{\mathrm{b}}$ & 0 & 0 & 0 \\
\hline
\end{tabular}

Note: ${ }^{\text {a }}$ verage enumeration of plates was less than $10 \mathrm{CFU} / \mathrm{mL}$ and enrichment tubes showed positive growth. ${ }^{\mathrm{b}}$ egative growth both on plates and tubes.

Table 4 Inactivation results of different UV on $V$. parahaemolyticus in artificial seawater

\begin{tabular}{cccccccc}
\hline Power & \multicolumn{3}{c}{$10 \mathrm{~W}$} & \multicolumn{3}{c}{$15 \mathrm{~W}$} \\
\cline { 6 - 8 } Time & 3 min & $5 \min$ & $10 \min$ & 1 min & 3 min & 5 min \\
\hline $\begin{array}{c}\text { Survivals } \\
/ \log \mathrm{CFU} \cdot \mathrm{mL}^{-1}\end{array}$ & $<1^{\mathrm{a}}$ & $<1$ & $0^{\mathrm{b}}$ & $<1$ & 0 & 0
\end{tabular}

Note: ${ }^{\text {a }}$ verage enumeration of plates was less than $10 \mathrm{CFU} / \mathrm{mL}$ and enrichment tubes showed positive growth. ${ }^{\mathrm{b}}$ egative growth both on plates and tubes.

\section{Conclusions}

SAEW has high bactericidal efficacy on $V$. parahaemolyticus and the higher the ACC, the shorter time it takes. The performance of SAEW is promising against $V$. parahaemolyticus inoculated in artificial seawater. With an appropriate ratio about $1: 15$, SAEW inactivated $V$. parahaemolyticus to undetectable level in artificial seawater. The bacterial destruction effect of SAEW was different at different ACC levels and within or without organic condition. Therefore, we can choose relatively high ACC of SAEW to reduce the time, or under some chlorine rigorous conditions we can use low ACC of SAEW and prolong the inactivating time. More research should be done for the further use of SAEW as an additive in aquaculture industry, for example, the sanitization potential against other foodborne pathogens in aquaculture seawater and efficiency under dynamic hybrid condition.

\section{Acknowledgements}

This research was financially supported by the National Key R\&D Program of China (Project number: 2018YFD0701001), the Key Laboratory of Equipment and Informatization in Environment Controlled Agriculture, Ministry of Agriculture, P.R. China and the Shanghai Sailing Program (19YF1443500).

\section{[References]}

[1] FAO. The State of the World Fisheries and Aquaculture. in: Tech. Rep., Food and Agriculture Organization of the United Nations, Rome, 2018; pp.2-7.

[2] Sani N A, Ariyawansa S, Babji A S, Hashim J K. The risk assessment of Vibrio parahaemolyticus in cooked black tiger shrimp (Penaeus monodon) in Malaysia. Food Control, 2013; 31(2): 546-552.

[3] Daniels N A, MacKinnon L, Bishop R, Altekruse S, Ray B, Hammond R M. Vibrio parahaemolyticus infections in the United States 1973-1998. J Infect Dis, 2000; 181(5): 1661-1666.

[4] Park S Y, Ha S D. Reduction of Escherichia coli and Vibrio parahaemolyticus counts on freshly sliced shad (Konosirus punctatus) by combined treatment of slightly acidic electrolyzed water and ultrasound using response surface methodology. Food Bioprocess Tech, 2015; 8(8): 1762-1770.

[5] Torlak E. Inactivation of Alicyclobacillus acidoterrestris spores in aqueous suspension and on apples by neutral electrolyzed water. Int $\mathrm{J}$ Food Microbiol, 2014; 185: 69-72.

[6] Koide S, Takeda J, Shi J, Shono H, Atungulu G G. Disinfection efficacy of slightly acidic electrolyzed water on fresh cut cabbage. Food Control, 2009; 20(3): 294-297.

[7] Issa-Zacharia A, Kamitani Y, Miwa N, Muhimbula H, Iwasaki K Application of slightly acidic electrolyzed water as a potential non-thermal food sanitizer for decontamination of fresh ready-to-eat vegetables and sprouts. Food Control, 2011; 22(3-4): 601-607.

[8] Izumi H. Electrolyzed water as a disinfectant for fresh-cut vegetables. J Food Sci, 1999; 64(3): 536-539.

[9] Quan Y, Choi K D, Chung D, Shin I S. Evaluation of bactericidal activity of weakly acidic electrolyzed water (WAEW) against Vibrio vulnificus and Vibrio parahaemolyticus. Int J Food Microbiol, 2010; 136(3): 255-260.

[10] Issa-Zacharia A, Kamitani Y, Tiisekwa A, Morita K, Iwasaki K. In vitro inactivation of Escherichia coli, Staphylococcus aureus and Salmonella spp. using slightly acidic electrolyzed water. J Biosci Bioeng, 2010; 110(3): 308-313.

[11] Kim H J, Tango C N, Chelliah R, Oh D H. Sanitization efficacy of slightly acidic electrolyzed water against pure cultures of Escherichia coli, Salmonella enterica, Typhimurium, Staphylococcus aureus and Bacillus cereus spores, in comparison with different water hardness. Sci REP-UK, 2019; 9(1): 43-48.

[12] Ye Z, Wang S, Gao W, Nan S, Zhu S, He H, et al. Inactivation mechanism and apoptotic-like changes in Aeromonas hydrophila induced by slightly acidic electrolyzed water in freshwater. Transactions of the ASABE, 2018; 61(1): 305-314.

[13] Mansur A R, Wang J, Park M S, Oh D H. Growth model of Escherichia coli $\mathrm{O} 157: \mathrm{H} 7$ at various storage temperatures on Kale treated by thermosonication combined with slightly acidic electrolyzed water. J Food Protect, 2014; 77(1): 23-31.

[14] Hao J X, Qiu S, Li H Y, Chen T P, Liu H J, Li L T. Roles of hydroxyl radicals in electrolyzed oxidizing water (EOW) for the inactivation of Escherichia coli. Int J Food Microbiol, 2012; 155(3): 99-104.

[15] Guentzel,J L, Liang L K, Callan M A, Emmons S A, Dunham V L. Reduction of bacteria on spinach, lettuce, and surfaces in food service areas using neutral electrolyzed oxidizing water. Food Microbiol, 2008; 25(1): 36- 41.

[16] Kasai H, Kawana K, Labaiden M, Namba K, Yoshimizu M. Elimination of Escherichia coli from oysters using electrolyzed seawater. Aquaculture, 2011; 319(3-4): 315-318.

[17] Jorquera M A, Valencia G, Eguchi M, Katayose M, Riquelme C. Disinfection of seawater for hatchery aquaculture systems using electrolytic water treatment. Aquaculture, 2002; 207(3-4): 213-22.

[18] Katayose M., Yoshida K, Achiwa N, Eguchi M. Safety of electrolyzed seawater for use in aquaculture. Aquaculture, 2007; 264(1-4): 119-129.

[19] Wang J J, Sun W S, Jin M T, Liu H Q, Zhang W, Sun X H, et al. Fate of Vibrio parahaemolyticus on shrimp after acidic electrolyzed water treatment. Int J Food Microbiol, 2014; 179: 50-56.

[20] Berges J A, Franklin D J, Harrison P J. Evolution of an artificial seawater medium Improvements in enriched seawater, artificial water over the last two decades. J Phycol, 2001; 37(6): 1138-1145.

[21] Huang Y R, Hung Y C, Hsu S Y, Huang Y W, Hwang D F. Application of electrolyzed water in the food industry. Food Control, 2008; 19(4): 329-345.

[22] Waters B W, Hung Y. The effect of $\mathrm{pH}$ and chloride concentration on the stability and antimicrobial activity of chlorine-based sanitizers. J Food Sci, 2014; 79(4): 622-627.

[23] Rahman S M E, Ding T, Oh D H. Inactivation effect of newly developed low concentration electrolyzed water and other sanitizers against microorganisms on spinach. Food Control, 2010; 21(10): 1383-1387.

[24] McPherson L L. Understanding ORP's in the disinfection process. Water Eng. Manag, 1993; 140(11): 29-31.

[25] Jadeja R, Hung Y C, Bosilevac J M. Resistance of various shiga toxin-producing Escherichia coli to electrolyzed oxidizing water. Food Control, 2013; 30(2): 580-584.

[26] Oomori T, Oka T, Inuta T, Arata Y. The efficiency of disinfection of acidic electrolyzed water in the presence of organic materials. Anal Sci, 2000; 16(4): 365-369. 